\title{
An explicit solution for optimal investment in Heston model*
}

\author{
Elena Boguslavskaya ${ }^{\dagger \ddagger}$ \\ Brunel University London, United Kingdom, \\ Dmitry Muravey
Internatonal Laboratory of Quantitative Finance, \\ Higher School of Economics, Moscow, Russia
}

\begin{abstract}
In this paper we consider a variation of the Merton's problem with added stochastic volatility and finite time horizon. It is known that the corresponding optimal control problem may be reduced to a linear parabolic boundary problem under some assumptions on the underlying process and the utility function. The resulting parabolic PDE is often quite difficult to solve, even when it is linear. The present paper contributes to the pool of explicit solutions for stochastic optimal control problems. Our main result is the exact solution for optimal investment in Heston model.
\end{abstract}

\section{Introduction}

The optimal control theory for stochastic processes plays a prominent role in financial mathematics and allows to formulate and solve problems in optimal investment, optimal trading, and other areas related to finance. The seminal paper by Robert Merton [1] was generalized in several directions. The subsequent research has introduced more realistic and complex asset dynamics [2], 3], and more realistic market models [4. Similar mathematical problems arise in algorithmic trading [5, and market microstructure studies 6 .

In this paper we consider a variation of the Merton's problem with stochastic volatility and finite time horizon. As shown in [2, the optimal control problem may be reduced to a linear parabolic boundary problem under some assumptions on the underlying process and the utility function. Generally, due to the lack of solution smoothness for this boundary problem, one needs to find a viscosity solution to a non-linear equation.

The resulting parabolic PDE is often quite difficult to solve, even when it is linear. In several special cases the explicit solutions were obtained, see [7. for the model similar to Heston's, and [8] for Chacko-Viceira model.

The present paper contributes to the pool of explicit solutions for stochastic optimal control problems. Our main result is the exact solution to the optimal control problem within the framework of the celebrated Heston model 9 . The method of affine representations, as used in [7, cannot be applied here. To obtain the desired explicit solution we employ the theory of integral representations and special functions. Quasi-analytical solutions were obtained by asymptotic and perturbation methods in $[3$ for optimal control of more general processes.

\footnotetext{
${ }^{*}$ The research on this paper was supported by grant 14.12.31.2007 of the Russian government.

†elena@boguslavsky.net

${ }^{\ddagger}$ The author is supported by Daphne Jackson fellowship funded by ESPRC

§dmuravey@hse.ru
} 
The paper is organized as follows: in section 2 we formulate the problem; in section 3 we discuss the results of 2 on the representation of Bellman function as the solution of the linear parabolic equation, and extend their results to the case of exponential utility; in section 4 we present the main results of the paper; in section [5] we analyze the obtained explicit solution; in Appendix (section [6) we provide some technical asymptotics relations.

\section{Formulation of the problem}

Let $(X, V)=\left(X_{s}, V_{s}\right)_{s \geq t}$ be a vector stochastic process, given by the triangular system of stochastic differential equations

$$
\begin{aligned}
d X / X & =\mu d t+\sqrt{V} d B^{1}, \quad X_{t}=x \\
d V & =k(\Theta-V) d s+\sigma \sqrt{V} d B^{2}, \quad V_{t}=v
\end{aligned}
$$

where $B^{1}=\left(B_{t}^{1}\right)_{s \geq t} \quad B^{2}=\left(B_{t}^{2}\right)_{s \geq t}$ are correlated Wiener processes with correlation coefficient $\rho$, i.e. $\left\langle B^{1}, B^{2}\right\rangle_{t}=\rho t$, and $\mu, k, \Theta, \sigma$ are some constants. The model for the asset price $X$ defined by (2.1) is very well known in financial mathematics, and is called the Heston model [9].

In order to solve the system of SDE's (2.1) one needs to solve the second equation first to obtain $V$, and then solve for $X$. Process $V=\left(V_{s}\right)_{s \geq t}$ represents the volatility of asset $X$. Process $V$ is a Feller process, also called a CIR (Cox-Ingersoll-Ross) process in financial mathematics [10] - We assume that the Feller condition $2 k \Theta>\sigma^{2}$ is satisfied for $V$. Thus, $V$ is always positive given $V_{0}>0$.

Let us consider a controlled process $W=\left(W_{s}\right)_{s \geq t}$ given by

$$
d W=\alpha d X, \quad W_{t}=w .
$$

where control $\alpha=\left(\alpha_{s}\right)_{s \geq t}$ is adapted to filtration $\mathcal{F}_{\geq t}=\sigma\left\{B_{s}-B_{t}, s \geq t\right\}$ and $\int_{t}^{T} \alpha_{s}^{2} d t<\infty$.

Our aim is to find the control on which functional $\mathbf{E} U\left(W_{T}\right)$ achieves its maximum

$$
J(w, x, v, t)=\sup _{\alpha} \mathbf{E} U\left(W_{T}\right) .
$$

Function $J(w, x, v, t)$ is the Bellman function, which is a solution to HJB ( Hamilton-JacobiBellman) equation. Utility function $U(w)$ can be a power or an exponential utility function. For the convenience of notation we will omit the indices.

$$
U_{P}(w)=\frac{w^{\gamma}}{\gamma}, \quad \gamma<0, \quad U_{E}(w)=1-\frac{e^{-c w}}{c}, \quad c>0 .
$$

Note, that the logarithmic utility is a special case of the power utility with $\gamma=0$. This well known observation can be checked by taking the $\operatorname{limit}_{\gamma \rightarrow 0}\left(\lim ^{\gamma}-1\right) / \gamma=\log (w)$ and noting that utility functions are defined up to an additive constant.

The financial interpretation of the processes above is the following: process $W$ represents the wealth dynamics of the investor, while control $\alpha$ is the evolution of the investor's position in the asset. The utility function $U$ represents the investor's preferences. Thus, to invest optimally, one should find the optimal investment rule at each time $t$ such that the expected utility of the terminal wealth at time $T$ is maximised (2.3).

Function $J(w, x, v, t)$ is the solution of Hamilton-Jacobi-Bellman (HJB) equaion. The solution of HJB equation in the general case is a non-linear PDE. However, this equation may become linear under some choice of utility functions. Zariphopoulou [2] has proposed a method based on viscosity solution technique. Under this method the equation (2.3) may be reduced to a linear parabolic equation. The method may be applied to systems of stochastic differential equations of "triangular type". Under this restriction the drift and the diffusion of the underlying asset $X$ may depend on the volatility process $V$ in arbitrary way. 
It is much easier to perform qualitative and quantitative analysis of the optimal solution while provided with the exact formula. Nevertheless, it is not always possible to find an explicit solution to a linear parabolic PDE. Explicit solutions were obtained in some special cases: see [7] for the model similar to Heston's, and [8] for Chacko-Viceira model. Either of the above results are based on affine representations of solutions. This technique is not applicable in the model we consider (2.1).

In the present paper we propose a method based on Laplace transform. This technique allows us to obtain an explicit solution in terms of confluent hypergeometric functions.

\section{T. Zariphopoulou's result and extensions}

A quite general model for optimal investment (2.3) with power utility function $U_{P}$ was considered in 2. The asset price dynamics $X$ dependent on random process $V$ was given by the triangular system of stochastic differential equations

$$
\begin{aligned}
d X / X & =\mu(V, t) d t+\sigma(V, t) d B^{1} \\
d V & =b(V, t) d t+a(V, t) d B^{2}
\end{aligned}
$$

where $B^{1}=\left(B_{t}^{1}\right)_{s \geq t} \quad B^{2}=\left(B_{t}^{2}\right)_{s \geq t}$ are correlated Wiener processes with correlation coefficient $\rho$.

We assume the necessary restrictions on the coefficients of system (3.1) being satisfied in order to guarantee the existence of a strong solution $(X, V)$.

Theorem 3.1 (Zariphopoulou). For process (3.1) and power utility function 2.4 we have the following representation of Bellman function (2.3)

$$
J_{P}(w, x, v, t)=\frac{w^{\gamma}}{\gamma} f^{1 / \delta}(v, t)
$$

where

$$
\delta=1+\rho^{2} \frac{\gamma}{1-\gamma}
$$

and function $f=(v, t)$ is a solution to the following parabolic boundary value problem

$$
\begin{array}{r}
f_{t}+\frac{1}{2} a^{2}(v, t) f_{v v}+\left(b(v, t)+\rho \frac{\gamma \mu(v, t) a(v, t)}{(1-\gamma) \sigma(v, t)}\right) f_{v}+\frac{\gamma}{1-\gamma} \frac{\delta}{2} \frac{\mu^{2}(v, t)}{\sigma^{2}(v, t)} f=0 \\
f(v, T)=1 .
\end{array}
$$

The optimal synthesis is given by

$$
\alpha_{P}^{*}(w, x, v, t)=\frac{w}{x(1-\gamma)}\left(\frac{\mu(v, t)}{\sigma^{2}(v, t)}+\frac{\rho}{\delta} \frac{a(v, t)}{\sigma(v, t)} \frac{f_{v}(v, t)}{f(v, t)}\right) .
$$

To find the optimal control at time $t$ one should substitute the values for processes $W$ and $(X, V)$ into synthesis (3.5). Note, that representation (3.2) can be explained as follows. Firstly, one can separate variables in the Bellman function $J(w, x, v, t)=u(w) R(x, v, t)$. This trick is well known for the power utility case. Secondly, from the form of the coefficients and the boundary condition, one can see that the solution does not depend on variable $x$. Thirdly, after the above manipulations, the remaining non-linear equation can be linearised by substitution $R^{\delta}(v, t)=f(v, t)$.

We use similar reasoning as above to extend Zariphopoulou's formula to the case of exponential utility. It is our first result in this paper. 
Proposition 3.1. Under exponential utility function $U_{E}$, the Bellman function (2.3) is represented by

$$
J_{E}(x, w, v, t)=1-\frac{e^{-c w}}{c} f^{1 / \delta}(v, t)
$$

where

$$
\delta=1-\rho^{2},
$$

and function $f=f(v, t)$ is a solution to the following parabolic boundary value problem

$$
\begin{aligned}
f_{t}+\frac{1}{2} a^{2}(v, t) f_{v v}+\left(b(v, t)-\rho \frac{\mu(v, t) a(v, t)}{\sigma(v, t)}\right) f_{v}-\frac{\delta}{2} \frac{\mu^{2}(v, t)}{\sigma^{2}(v, t)} f & =0, \\
f(v, T) & =1 .
\end{aligned}
$$

The optimal synthesis is given by

$$
\alpha_{E}^{*}(w, x, v, t)=\frac{1}{c x}\left(\frac{\mu(v, t)}{\sigma^{2}(v, t)}+\frac{\rho}{\delta} \frac{a(v, t)}{\sigma(v, t)} \frac{f_{v}(v, t)}{f(v, t)}\right) .
$$

Note, that constant $\delta$ given by (3.7) and equation (3.8) for $f$ can be obtained from power utility formula (3.3) and (3.4) by taking limit $\gamma \rightarrow-\infty$.

\section{The main result}

Let us return to the original problem. Consider the Heston model given by (2.1). Using the results from section 3, in particular substitutions (3.2) and (3.6) from Theorem 3.1 and Proposition 3.1 respectively, we can reduce the original boundary value problem for Bellman function to the following boundary value problem for function $f=f(v, t)$

$$
\begin{aligned}
\frac{\sigma^{2} v}{2} f_{v v}+\left(k \Theta-\frac{1-\delta}{\rho} \mu \sigma-k v\right) f_{v}-\frac{C}{v} f+f_{t} & =0 . \\
f(v, T) & =1,
\end{aligned}
$$

Equation (4.1) is the same for both cases of power and exponential utility functions, with the only difference in the values of constants $\delta$ and $C$. In the case of power utility function

$$
\delta=1+\rho^{2} \frac{\gamma}{1-\gamma}, \quad C=-\frac{\gamma}{1-\gamma} \frac{\mu^{2}}{2} \delta
$$

For the exponential utility case

$$
\delta=1-\rho^{2}, \quad C=\frac{\mu^{2}}{2} \delta .
$$

Lemma 4.1. The solution of 4.1] is given by

$$
f(v, t)=\frac{\Gamma(\eta-\lambda+1 / 2)}{\Gamma(2 \eta+1)} e^{-\Psi(v, t) / 2}(\Psi(v, t))^{\lambda} M_{\lambda, \eta}(\Psi(v, t)),
$$

where

$$
\Psi(v, t)=\frac{2 k v}{\sigma^{2}\left(e^{k(T-t)}-1\right)}, \quad \lambda=-\frac{k \Theta}{\sigma^{2}}+\frac{(1-\delta) \mu}{\rho \sigma}, \quad \eta=\sqrt{\left(\lambda+\frac{1}{2}\right)^{2}+\frac{2 C}{\sigma^{2}}},
$$

$M_{\lambda, \eta}(z)$ is a Whittaker's function, and $\Gamma(z)$ is a Gamma function.

Moreover, we have

$$
\frac{f_{v}}{f}=\frac{(\eta+\lambda+1 / 2)}{v} \frac{M_{1+\lambda, \eta}(\Psi(v, t))}{M_{\lambda, \eta}(\Psi(v, t))} .
$$


Proof. Making substitutions

$$
\tilde{v}=\frac{2 k v}{\sigma^{2}}, \quad k(T-t)=\tilde{\tau}, \quad f(v, t)=e^{-\lambda \tilde{\tau}} \tilde{v}^{\lambda} e^{\tilde{v} / 2} h(\tilde{v}, \tilde{\tau})
$$

we get the following boundary value problem for $h$

$$
\begin{array}{r}
h_{\tilde{v} \tilde{v}}+\left(-\frac{1}{4}+\frac{1 / 4-\eta^{2}}{\tilde{v}^{2}}\right) h=\frac{1}{\tilde{v}} h_{\tilde{\tau}} . \\
h(\tilde{v}, 0)=\tilde{v}^{-\lambda} e^{-\tilde{v} / 2} .
\end{array}
$$

Let $G(\tilde{v} ; \zeta)$ be the Laplace transform of function $h(\tilde{v}, \tilde{\tau})$ with respect to $\tilde{\tau}$

$$
G(\tilde{v} ; \zeta)=\int_{0}^{\infty} e^{\zeta \tilde{\tau}} h(\tilde{v}, \tilde{\tau}) d \tilde{\tau}
$$

By denoting $\chi(\tilde{v})=\tilde{v}^{-1-\lambda} e^{-\tilde{v} / 2}$, one can see that

$$
G^{\prime \prime}+\left(-\frac{1}{4}-\frac{\zeta}{\tilde{v}}+\frac{1 / 4-\eta^{2}}{\tilde{v}^{2}}\right) G=-\chi(\tilde{v}) .
$$

If made homogeneous, equation (4.8) is a Whittaker equation. Whittaker functions $M_{-\zeta, \eta}(\tilde{v})$ and $W_{-\zeta, \eta}(\tilde{v})$ are two linearly independent solutions of a Whittaker equation. One can check by substitution that the formula below is the solution of equation (4.8)

$$
G(\tilde{v} ; \zeta)=\frac{\Gamma(1 / 2+\zeta+\eta)}{\Gamma(1+2 \eta)}\left(M_{-\zeta, \eta}(\tilde{v}) \int_{0}^{\tilde{v}} \chi(\varphi) W_{-\zeta, \eta}(\varphi) d \varphi+W_{-\zeta, \eta}(\tilde{v}) \int_{\tilde{v}}^{\infty} \chi(\varphi) M_{-\zeta, \eta}(\varphi) d \varphi\right) .
$$

To proceed further we use formula 6.669.4 from [12

$$
\begin{array}{r}
\int_{0}^{\infty} e^{-\frac{1}{2}\left(a_{1}+a_{2}\right) t \cosh x} \operatorname{coth}^{2 \nu}\left(\frac{1}{2} x\right) I_{2 \mu}\left(t \sqrt{a_{1} a_{2}} \sinh x\right) d x=\frac{\Gamma\left(\frac{1}{2}+\mu-\nu\right)}{t \sqrt{a_{1} a_{2}} \Gamma(1+2 \mu)} W_{\nu, \mu}\left(a_{1} t\right) M_{\nu, \mu}\left(a_{2} t\right), \\
\operatorname{Re}\left(\frac{1}{2}+\mu-\nu\right)>0, \quad \operatorname{Re} \mu>0, \quad a_{1}>a_{2} .
\end{array}
$$

The above formula from [12] allows us to rewrite (4.9) as

$$
G(\tilde{v} ; \zeta)=\sqrt{\tilde{v}} \int_{0}^{\infty} \int_{0}^{\infty} \varphi^{-1 / 2-\lambda} e^{-\frac{\varphi}{2}-\frac{\tilde{v}+\varphi}{2} \cosh \psi} \tanh ^{2 \zeta}\left(\frac{\psi}{2}\right) I_{2 \eta}(\sqrt{\tilde{v} \varphi} \sinh \psi) d \varphi d \psi
$$

Applying 6.643.2 from [12]

$$
\int_{0}^{\infty} x^{\mu-\frac{1}{2}} e^{-\alpha x} I_{2 \nu}(2 \beta \sqrt{x}) d x=\frac{\Gamma\left(\mu+\nu+\frac{1}{2}\right)}{\Gamma(2 \nu+1)} \beta^{-1} e^{\frac{\beta^{2}}{2 \alpha}} \alpha^{-\mu} M_{-\mu, \nu}\left(\frac{\beta^{2}}{\alpha}\right), \quad \operatorname{Re}\left(\mu+\nu+\frac{1}{2}\right)>0
$$

to the internal integral in (4.10), we obtain

$$
G(\tilde{v} ; \zeta)=e^{-\tilde{v} / 2} \frac{\Gamma(\eta-\lambda+1 / 2)}{\Gamma(1+2 \eta)} \int_{0}^{\infty} e^{(1-\cosh \psi) \tilde{v} / 4} \tanh ^{2 \zeta}\left(\frac{\psi}{2}\right)\left(\frac{\cosh \psi+1}{2}\right)^{\lambda} M_{\lambda, \eta}\left(\frac{\cosh \psi-1}{2} \tilde{v}\right) \frac{2 d \psi}{\sinh \psi} .
$$

By changing the variable of integration $\psi$ in (4.12) as

$$
2 \log \left[\tanh \left(\frac{\psi}{2}\right)\right]=\nu, \quad \frac{2 d \psi}{\sinh \psi}=d \nu, \quad \cosh \psi-1=\frac{2 e^{\nu}}{1-e^{\nu}}
$$


we get

$$
G(\tilde{v} ; \zeta)=e^{-\tilde{v} / 2} \frac{\Gamma(\eta-\lambda+1 / 2)}{\Gamma(1+2 \eta)} \int_{-\infty}^{0} \exp \left\{-\frac{\tilde{v}}{2} \frac{e^{\nu}}{e^{\nu}-1}+\zeta \nu\right\}\left(\frac{1}{e^{\nu}-1}\right)^{\lambda} M_{\lambda, \eta}\left(\tilde{v} \frac{e^{\nu}}{e^{\nu}-1}\right) d \nu .
$$

Inverting the Laplace transform, and using substitutions (4.7), we recover the formula for $f(v, t)$

$$
f(v, t)=\frac{1}{2 \pi i} \frac{\Gamma(\eta-\lambda+1 / 2)}{\Gamma(1+2 \eta)} \int_{N-i \infty}^{N+i \infty} \int_{-\infty}^{0} \exp \left\{-\frac{\tilde{v}}{2} \frac{e^{\nu}}{e^{\nu}-1}\right\} e^{\zeta(\nu+\tilde{\tau})}\left(\tilde{v} \frac{e^{-\tilde{\tau}}}{e^{\nu}-1}\right)^{\lambda} M_{\lambda, \eta}\left(\tilde{v} \frac{e^{\nu}}{e^{\nu}-1}\right) d \zeta d \nu
$$

where $N$ is a number such that all residues of the integrand are to the right of it.

Using the well-known representation of Dirac function

$$
\frac{1}{2 \pi i} \int_{N-i \infty}^{N+i \infty} e^{z \zeta} d \zeta=\delta(z)
$$

and changing the order of integration in (4.13), we get

$$
f(v, t)=\frac{\Gamma(\eta-\lambda+1 / 2)}{\Gamma(1+2 \eta)} \int_{-\infty}^{0} \delta(\nu+\tilde{\tau}) \exp \left\{-\frac{\tilde{v}}{2} \frac{e^{\nu}}{e^{\nu}-1}\right\}\left(\tilde{v} \frac{e^{-\tilde{\tau}}}{e^{\nu}-1}\right)^{\lambda} M_{\lambda, \eta}\left(\tilde{v} \frac{e^{\nu}}{e^{\nu}-1}\right) d \nu
$$

Note, that $\tilde{\tau} \geq 0$. Thus, we can complement the range of integration in (4.14) to the whole line, and, using the definition of Dirac's function, namely $\int_{-\infty}^{\infty} \delta(\zeta-z) g(\zeta) d \zeta=g(z)$ for any continuous $g$, we get

$$
f(v, t)=\frac{\Gamma(\eta-\lambda+1 / 2)}{\Gamma(1+2 \eta)} \exp \left\{-\frac{\tilde{v}}{2} \frac{e^{\tilde{\tau}}}{e^{\tilde{\tau}}-1}\right\}\left(\tilde{v} \frac{e^{-\tilde{\tau}}}{e^{\tilde{\tau}}-1}\right)^{\lambda} M_{\lambda, \eta}\left(\tilde{v} \frac{e^{\tilde{\tau}}}{e^{\tilde{\tau}}-1}\right) .
$$

Finally, by reversing the change of variables (4.7), we receive the main formula (4.4). The expression (4.6) for $f_{v} / f$ is obtained by using differential rules for Whittaker functions (see [11]). $\triangle$

The following theorem is the analogue of the results from section 3 but for Heston's model. It gives an exact solution for the optimal control and Bellman function.

Theorem 4.1. For power utility 2.4) and process (2.1) the Bellman function (2.3) is given by

$$
J_{P}(w, x, v, t)=\frac{w^{\gamma}}{\gamma} f^{1 / \delta}(v, t)
$$

and the optimal control is

$$
\alpha_{P}^{*}(w, x, v, t)=\frac{w}{x(1-\gamma)}\left(\frac{\mu}{v}+\frac{\rho \sigma}{\delta} \frac{(\eta+\lambda+1 / 2)}{v} \frac{M_{1+\lambda, \eta}(\Psi(v, t))}{M_{\lambda, \eta}(\Psi(v, t))}\right),
$$

where

$$
\begin{aligned}
f(v, t) & =\frac{\Gamma(\eta-\lambda+1 / 2)}{\Gamma(2 \eta+1)} e^{-\Psi(v, t) / 2}(\Psi(v, t))^{\lambda} M_{\lambda, \eta}(\Psi(v, t)), \\
\delta & =1+\rho^{2} \frac{\gamma}{1-\gamma}, \quad \lambda=-\frac{k \Theta}{\sigma^{2}}+\frac{(1-\delta) \mu}{\rho \sigma}, \quad C=-\frac{\gamma}{1-\gamma} \frac{\mu^{2}}{2} \delta, \quad \eta=\sqrt{\left(\lambda+\frac{1}{2}\right)^{2}+\frac{2 C}{\sigma^{2}}}, \\
\Psi(v, t) & =\frac{2 k v}{\sigma^{2}\left(e^{k(T-t)}-1\right)},
\end{aligned}
$$

$M_{\lambda, \eta}(z)$ is a Whittaker's function, and $\Gamma(z)$ is a gamma function. 
Theorem 4.2. For exponential utility 2.4) and process (2.1) The Bellman function is given by

$$
J_{E}(x, w, v, t)=1-\frac{e^{-c w}}{c} f^{1 / \delta}(v, t)
$$

and the optimal control is

$$
\alpha_{E}^{*}(w, x, v, t)=\frac{1}{c x}\left(\frac{\mu}{v}+\frac{\rho \sigma}{\delta} \frac{(\eta+\lambda+1 / 2)}{v} \frac{M_{1+\lambda, \eta}(\Psi(v, t))}{M_{\lambda, \eta}(\Psi(v, t))}\right),
$$

where

$$
\begin{aligned}
f(v, t) & =\frac{\Gamma(\eta-\lambda+1 / 2)}{\Gamma(2 \eta+1)} e^{-\Psi(v, t) / 2}(\Psi(v, t))^{\lambda} M_{\lambda, \eta}(\Psi(v, t)), \\
\delta & =1-\rho^{2}, \quad \lambda=-\frac{k \Theta}{\sigma^{2}}+\frac{(1-\delta) \mu}{\rho \sigma}, \quad C=\frac{\mu^{2}}{2} \delta, \quad \eta=\sqrt{\left(\lambda+\frac{1}{2}\right)^{2}+\frac{2 C}{\sigma^{2}}}, \\
\Psi(v, t) & =\frac{2 k v}{\sigma^{2}\left(e^{k(T-t)}-1\right)},
\end{aligned}
$$

$M_{\lambda, \eta}(z)$ is a Whittaker's function, and $\Gamma(z)$ is a gamma function.

Comment. The model (3.1) with drift $\mu\left(X_{t}, t\right)=V_{t}$ is studied in [7]. For that particular model the coefficient for $f$ in (3.4) is proportional to $v$. This is the reason why the affine representation is possible in that specific case. Moreover, if the boundary condition is not constant, the affine representation is not possible again. Our method, on the contrary, allows us to obtain the required solution for any boundary conditions and for both models, either Heston's or the model considered in 7 .

\section{Analysis}

\subsection{Analysis of the optimal control}

Optimal control in (4.17) and (4.19) is proportional to the sum of two terms: the first term $\mu / v$ corresponds to the static portfolio optimization problem. It it simply the ratio of the instantaneous drift to the instantaneous variance and does not depend on time or on the parameters of the volatility process. The second term

$$
\frac{\rho \sigma}{\delta} \frac{(\eta+\lambda+1 / 2)}{v} \frac{M_{1+\lambda, \eta}(\Psi(v, t))}{M_{\lambda, \eta}(\Psi(v, t))}
$$

represents the hedging of the future opportunity set ( see Merton [14]). This term vanishes in a number of important special cases:

$\rho=0$ : no correlation between the two driving Brownian motions,

$\gamma=0$ : when the investor has log-utility.

The case of zero volatility of volatility limit of Heston model ( $\sigma=0$ in (2.1) $)$ turns the asset process into a process similar to a geometric Brownian motion but with a deterministically time varying volatility. If in addition we set $V_{0}=\Theta$ in (2.1), then we get a geometric Brownian motion.

Using the asymptotic results from Appendix 6 in case $\sigma \sim 0$, we get

$$
f(v, t) \sim 1, \quad \frac{f_{v}(v, t)}{f(v, t)} \sim \frac{C}{k v^{2}} e^{k(T-t)} .
$$


Therefore, for $\sigma \sim 0$ the Bellman function is approximately equal to the utility function, namely $J_{P} \sim U_{P}, J_{E} \sim U_{E}$; and the optimal controls are

$$
\begin{aligned}
& \alpha_{P}^{*}(w, x, v, t)=\frac{w}{x(1-\gamma)}\left(\frac{\mu}{v}+\frac{\rho \sigma}{\delta_{P}} \frac{C_{P}}{k v^{2}} e^{k(T-t)}\right), \\
& \alpha_{E}^{*}(w, x, v, t)=\frac{1}{c x}\left(\frac{\mu}{v}+\frac{\rho \sigma}{\delta_{E}} \frac{C_{E}}{k v^{2}} e^{k(T-t)}\right),
\end{aligned}
$$

where constants $C_{[-]}$and $\delta_{[-]}$depend on the choice of utility.

\subsection{Hedging interpretation via a bond}

Suppose $C$ is given by (4.2) or (4.3). With substitution $C / v=r, f(v, t)=g(r, t)$ we can rewrite (4.1) as

$$
\begin{array}{r}
\frac{b^{2} r^{3}}{2} g_{r r}+h r(m-r) g_{r}-r g+g_{t}=0, \\
g(r, T)=1,
\end{array}
$$

where

$$
b=\frac{\sigma}{\sqrt{C}}, \quad h=-\frac{\sigma^{2}}{C}(1+\lambda), \quad m=-\frac{k C}{\sigma^{2}(1+\lambda)} .
$$

Using the Feynman-Kac formula we get the following expression for $g=g(r, t)$

$$
g(r, t)=\mathbf{E} e^{-\int_{t}^{T} r_{s} d s}
$$

where process $r=\left(r_{s}\right)_{s \geq t}$ is a solution to the following stochastic differential equation

$$
d r=h r(m-r) d t+b r^{3 / 2} d B^{2}, \quad r_{t}=C / v_{t} .
$$

Equation (5.6) describes the well-known 3/2-model of stochastic interest rates (see [13].) In this model, if $r$ is interpreted as the short rate, value of a zero coupon bond is given by (5.6), see [15]. Hence, the ratio $f_{v} / f$ can be expressed as

$$
\frac{f_{v}(v, t)}{f(v, t)}=\frac{d}{d v} \log \mathbf{E}\left[e^{-\int_{t}^{T} r_{s} d s} \mid r_{t}=C / v\right] .
$$

Substituting (5.7) into formulae for optimal control (4.17) and (4.19) one can get an interpretation of the hedging of the future opportunity set via a bond.

\section{Appendix. Technical asymptotics relations}

It follows from (4.4), that function $f=f(v, t)$ can be written as a function of only $\Psi$, namely $f=f(\Psi)$, where $\Psi=\Psi(v, t)$ is given by (4.5). This representation allows us to study the asymptotics of Bellman function and the optimal control by using the asymptotics of Whittaker functions $M_{\lambda, \mu}(z)$ (see, for example, [11]).

Indeed, by using the well known formulae below

$$
M_{\lambda, \eta}(z) \sim z^{\eta+1 / 2}(1+O(z)), \quad z \rightarrow 0, \quad M_{\lambda, \eta}(z) \sim \frac{\Gamma(1+2 \eta)}{\Gamma(1 / 2-\lambda+\eta)} e^{z / 2} z^{-\lambda}, \quad z \rightarrow \infty,
$$

we get

$$
f(v, t) \sim \frac{\Gamma(1 / 2-\lambda+\eta)}{\Gamma(1+2 \eta)} \Psi^{\eta+1 / 2}(v, t)\left[\Psi^{\lambda}(v, t)+O(\Psi(v, t))\right], \quad \Psi(v, t) \rightarrow 0,
$$


and

$$
f(v, t) \sim 1, \quad \Psi(v, t) \rightarrow \infty .
$$

For $f_{v}(v, t) / f$ we have

$$
\frac{f_{v}(v, t)}{f(v, t)} \sim \frac{(\eta+\lambda+1 / 2)}{v}, \quad \Psi(v, t) \rightarrow 0,
$$

and

$$
\frac{f_{v}(v, t)}{f(v, t)} \sim \frac{2 C}{\sigma^{2}} \frac{1}{v \Psi(v, t)}, \quad \Psi(v, t) \rightarrow \infty .
$$

Acknowledgements. The authors are grateful to Yury Kabanov for fruitful discussions and useful suggestions.

\section{References}

[1] R.C. Merton (1971) Optimum consumption and portfolio rules in a continuous-time model. Journal of Economic theory. 3.4. 373-413.

[2] T. Zariphopoulou (2001) A solution approach to valuation with unhedgeable risks. Finance Stochast., 5, 61-82.

[3] J. Fouque, R. Sircar and T. Zariphopoulou (2013) Portfolio Optimization and Stochastic Volatility Asymptotics. Preprint

[4] Yu. Kabanov, M. Safarian (2009) Market with transaction costs. Mathematical theory. Springer-Verlag.

[5] M. Boguslavsky, E. Boguslavskaya (2003) Arbitrage under power RISK. 06. 49-53.

[6] A. Obizhaeva, J. Wang (2013) Optimal trading strategy and supply/demand dynamics. Journal of financial markets. 16. 1-32.

[7] H. Kraft (2005) Optimal portfolios and Heston's Stochastic volatility model: an explicit solution for power utility. Quantitative Finance. 5. 303-313.

[8] G. Chacko and L. Viceira (2005) Dynamic Consumption and portfolio choice with stochastic volatility in incomplete markets Review of Financial Studies, 18, 1369-1402.

[9] S. Heston (1993) A closed form solution for options with stochastic volatility with applications to bond and currency options. Review of Financial Studies, 6, 2, 327-343.

[10] J.C. Cox, J.E. Ingersoll, S.A. Ross (1985) A theory of the term structure of interest rates. Econometrica. 53.2.385-407.

[11] Abramowitz M., Stegun I., Eds. (1972) Handbook of mathematical functions with formulas, graphs and mathematical tables. Dover, New York.

[12] Gradshteyn I.S., Ryzhik I.M. (1980) Tables of integrals, series and products. Academic Press, New York.

[13] J. Gatheral, N. Taleb (2006) Volatility surface: a practioner guide. Wiley.

[14] R.C. Merton, Continuous-Time Finance, Blackwell Publishers, (1990).

[15] D. Brigo, F. Mercurio (2006) Interest rate models - theory and practice: with smile, inflation and credit. Springer finance. 


\title{
An explicit solution for optimal investment in Heston model*
}

\author{
Elena Boguslavskaya ${ }^{\dagger \ddagger}$ \\ Brunel University London, United Kingdom, \\ Dmitry Muravey
Internatonal Laboratory of Quantitative Finance, \\ Higher School of Economics, Moscow, Russia
}

\begin{abstract}
In this paper we consider a variation of the Merton's problem with added stochastic volatility and finite time horizon. It is known that the corresponding optimal control problem may be reduced to a linear parabolic boundary problem under some assumptions on the underlying process and the utility function. The resulting parabolic PDE is often quite difficult to solve, even when it is linear. The present paper contributes to the pool of explicit solutions for stochastic optimal control problems. Our main result is the exact solution for optimal investment in Heston model.
\end{abstract}

\section{Introduction}

The optimal control theory for stochastic processes plays a prominent role in financial mathematics and allows to formulate and solve problems in optimal investment, optimal trading, and other areas related to finance. The seminal paper by Robert Merton [12] was generalized in several directions. The subsequent research has introduced more realistic and complex asset dynamics [15, 6, and more realistic market models [10. Similar mathematical problems arise in algorithmic trading [2], and market microstructure studies [14].

In this paper we consider a variation of the Merton's problem with stochastic volatility and finite time horizon. As shown in [15, the optimal control problem may be reduced to a linear parabolic boundary problem under some assumptions on the underlying process and the utility function. Generally, due to the lack of solution smoothness for this boundary problem, one needs to find a viscosity solution to a non-linear equation.

The resulting parabolic PDE is often quite difficult to solve, even when it is linear. In several special cases the explicit solutions were obtained, see [11 for the model similar to Heston's, and [4] for Chacko-Viceira model.

The present paper contributes to the pool of explicit solutions for stochastic optimal control problems. Our main result is the exact solution to the optimal control problem within the framework of the celebrated Heston model [7. The method of affine representations, as used in [11, cannot be applied here. To obtain the desired explicit solution we employ the theory of integral representations and special functions. Quasi-analytical solutions were obtained by asymptotic and perturbation methods in [6] for optimal control of more general processes.

\footnotetext{
${ }^{*}$ The research on this paper was supported by grant 14.12.31.2007 of the Russian government.

†elena@boguslavsky.net

${ }^{\ddagger}$ The author is supported by Daphne Jackson fellowship funded by ESPRC

§dmuravey@hse.ru
} 
The paper is organized as follows: in section 2 we formulate the problem; in section 3 we discuss the results of 15 on the representation of Bellman function as the solution of the linear parabolic equation, and extend their results to the case of exponential utility; in section 4 we present the main results of the paper; in section [5] we analyze the obtained explicit solution; in Appendix (section [6) we provide some technical asymptotics relations.

\section{Formulation of the problem}

Let $(X, V)=\left(X_{s}, V_{s}\right)_{s \geq t}$ be a vector stochastic process, given by the triangular system of stochastic differential equations

$$
\begin{aligned}
d X / X & =\mu d t+\sqrt{V} d B^{1}, \quad X_{t}=x \\
d V & =k(\Theta-V) d s+\sigma \sqrt{V} d B^{2}, \quad V_{t}=v
\end{aligned}
$$

where $B^{1}=\left(B_{t}^{1}\right)_{s \geq t} \quad B^{2}=\left(B_{t}^{2}\right)_{s \geq t}$ are correlated Wiener processes with correlation coefficient $\rho$, i.e. $\left\langle B^{1}, B^{2}\right\rangle_{t}=\rho t$, and $\mu, k, \Theta, \sigma$ are some constants. The model for the asset price $X$ defined by (2.1) is very well known in financial mathematics, and is called the Heston model [7].

In order to solve the system of SDE's (2.1) one needs to solve the second equation first to obtain $V$, and then solve for $X$. Process $V=\left(V_{s}\right)_{s \geq t}$ represents the volatility of asset $X$. Process $V$ is a Feller process, also called a CIR (Cox-Ingersoll-Ross) process in financial mathematics [5] - We assume that the Feller condition $2 k \Theta>\sigma^{2}$ is satisfied for $V$. Thus, $V$ is always positive given $V_{0}>0$.

Let us consider a controlled process $W=\left(W_{s}\right)_{s \geq t}$ given by

$$
d W=\alpha d X, \quad W_{t}=w .
$$

where control $\alpha=\left(\alpha_{s}\right)_{s \geq t}$ is adapted to filtration $\mathcal{F}_{\geq t}=\sigma\left\{B_{s}-B_{t}, s \geq t\right\}$ and $\int_{t}^{T} \alpha_{s}^{2} d t<\infty$.

Our aim is to find the control on which functional $\mathbf{E} U\left(W_{T}\right)$ achieves its maximum

$$
J(w, x, v, t)=\sup _{\alpha} \mathbf{E} U\left(W_{T}\right)
$$

Function $J(w, x, v, t)$ is the Bellman function, which is a solution to HJB ( Hamilton-JacobiBellman) equation. Utility function $U(w)$ can be a power or an exponential utility function. For the convenience of notation we will omit the indices.

$$
U_{P}(w)=\frac{w^{\gamma}}{\gamma}, \quad \gamma<0, \quad U_{E}(w)=1-\frac{e^{-c w}}{c}, \quad c>0 .
$$

Note, that the logarithmic utility is a special case of the power utility with $\gamma=0$. This well known observation can be checked by taking the $\operatorname{limit}_{\gamma \rightarrow 0}\left(\lim ^{\gamma}-1\right) / \gamma=\log (w)$ and noting that utility functions are defined up to an additive constant.

The financial interpretation of the processes above is the following: process $W$ represents the wealth dynamics of the investor, while control $\alpha$ is the evolution of the investor's position in the asset. The utility function $U$ represents the investor's preferences. Thus, to invest optimally, one should find the optimal investment rule at each time $t$ such that the expected utility of the terminal wealth at time $T$ is maximised (2.3).

Function $J(w, x, v, t)$ is the solution of Hamilton-Jacobi-Bellman (HJB) equaion. The solution of HJB equation in the general case is a non-linear PDE. However, this equation may become linear under some choice of utility functions. Zariphopoulou [15] has proposed a method based on viscosity solution technique. Under this method the equation (2.3) may be reduced to a linear parabolic equation. The method may be applied to systems of stochastic differential equations of "triangular type". Under this restriction the drift and the diffusion of the underlying asset $X$ may depend on the volatility process $V$ in arbitrary way. 
It is much easier to perform qualitative and quantitative analysis of the optimal solution while provided with the exact formula. Nevertheless, it is not always possible to find an explicit solution to a linear parabolic PDE. Explicit solutions were obtained in some special cases: see [7] for the model similar to Heston's, and [8] for Chacko-Viceira model. Either of the above results are based on affine representations of solutions. This technique is not applicable in the model we consider (2.1).

In the present paper we propose a method based on Laplace transform. This technique allows us to obtain an explicit solution in terms of confluent hypergeometric functions.

\section{T. Zariphopoulou's result and extensions}

A quite general model for optimal investment (2.3) with power utility function $U_{P}$ was considered in [15. The asset price dynamics $X$ dependent on random process $V$ was given by the triangular system of stochastic differential equations

$$
\begin{aligned}
d X / X & =\mu(V, t) d t+\sigma(V, t) d B^{1} \\
d V & =b(V, t) d t+a(V, t) d B^{2}
\end{aligned}
$$

where $B^{1}=\left(B_{t}^{1}\right)_{s \geq t} \quad B^{2}=\left(B_{t}^{2}\right)_{s \geq t}$ are correlated Wiener processes with correlation coefficient $\rho$.

We assume the necessary restrictions on the coefficients of system (3.1) being satisfied in order to guarantee the existence of a strong solution $(X, V)$.

Theorem 3.1 (Zariphopoulou). For process (3.1) and power utility function 2.4 we have the following representation of Bellman function (2.3)

$$
J_{P}(w, x, v, t)=\frac{w^{\gamma}}{\gamma} f^{1 / \delta}(v, t)
$$

where

$$
\delta=1+\rho^{2} \frac{\gamma}{1-\gamma}
$$

and function $f=(v, t)$ is a solution to the following parabolic boundary value problem

$$
\begin{array}{r}
f_{t}+\frac{1}{2} a^{2}(v, t) f_{v v}+\left(b(v, t)+\rho \frac{\gamma \mu(v, t) a(v, t)}{(1-\gamma) \sigma(v, t)}\right) f_{v}+\frac{\gamma}{1-\gamma} \frac{\delta}{2} \frac{\mu^{2}(v, t)}{\sigma^{2}(v, t)} f=0 \\
f(v, T)=1 .
\end{array}
$$

The optimal synthesis is given by

$$
\alpha_{P}^{*}(w, x, v, t)=\frac{w}{x(1-\gamma)}\left(\frac{\mu(v, t)}{\sigma^{2}(v, t)}+\frac{\rho}{\delta} \frac{a(v, t)}{\sigma(v, t)} \frac{f_{v}(v, t)}{f(v, t)}\right) .
$$

To find the optimal control at time $t$ one should substitute the values for processes $W$ and $(X, V)$ into synthesis (3.5). Note, that representation (3.2) can be explained as follows. Firstly, one can separate variables in the Bellman function $J(w, x, v, t)=u(w) R(x, v, t)$. This trick is well known for the power utility case. Secondly, from the form of the coefficients and the boundary condition, one can see that the solution does not depend on variable $x$. Thirdly, after the above manipulations, the remaining non-linear equation can be linearised by substitution $R^{\delta}(v, t)=f(v, t)$.

We use similar reasoning as above to extend Zariphopoulou's formula to the case of exponential utility. It is our first result in this paper. 
Proposition 3.1. Under exponential utility function $U_{E}$, the Bellman function (2.3) is represented by

$$
J_{E}(x, w, v, t)=1-\frac{e^{-c w}}{c} f^{1 / \delta}(v, t)
$$

where

$$
\delta=1-\rho^{2},
$$

and function $f=f(v, t)$ is a solution to the following parabolic boundary value problem

$$
\begin{aligned}
f_{t}+\frac{1}{2} a^{2}(v, t) f_{v v}+\left(b(v, t)-\rho \frac{\mu(v, t) a(v, t)}{\sigma(v, t)}\right) f_{v}-\frac{\delta}{2} \frac{\mu^{2}(v, t)}{\sigma^{2}(v, t)} f & =0, \\
f(v, T) & =1 .
\end{aligned}
$$

The optimal synthesis is given by

$$
\alpha_{E}^{*}(w, x, v, t)=\frac{1}{c x}\left(\frac{\mu(v, t)}{\sigma^{2}(v, t)}+\frac{\rho}{\delta} \frac{a(v, t)}{\sigma(v, t)} \frac{f_{v}(v, t)}{f(v, t)}\right) .
$$

Note, that constant $\delta$ given by (3.7) and equation (3.8) for $f$ can be obtained from power utility formula (3.3) and (3.4) by taking limit $\gamma \rightarrow-\infty$.

\section{The main result}

Let us return to the original problem. Consider the Heston model given by (2.1). Using the results from section 3, in particular substitutions (3.2) and (3.6) from Theorem 3.1 and Proposition 3.1 respectively, we can reduce the original boundary value problem for Bellman function to the following boundary value problem for function $f=f(v, t)$

$$
\begin{aligned}
\frac{\sigma^{2} v}{2} f_{v v}+\left(k \Theta-\frac{1-\delta}{\rho} \mu \sigma-k v\right) f_{v}-\frac{C}{v} f+f_{t} & =0 . \\
f(v, T) & =1,
\end{aligned}
$$

Equation (4.1) is the same for both cases of power and exponential utility functions, with the only difference in the values of constants $\delta$ and $C$. In the case of power utility function

$$
\delta=1+\rho^{2} \frac{\gamma}{1-\gamma}, \quad C=-\frac{\gamma}{1-\gamma} \frac{\mu^{2}}{2} \delta
$$

For the exponential utility case

$$
\delta=1-\rho^{2}, \quad C=\frac{\mu^{2}}{2} \delta .
$$

Lemma 4.1. The solution of 4.1] is given by

$$
f(v, t)=\frac{\Gamma(\eta-\lambda+1 / 2)}{\Gamma(2 \eta+1)} e^{-\Psi(v, t) / 2}(\Psi(v, t))^{\lambda} M_{\lambda, \eta}(\Psi(v, t)),
$$

where

$$
\Psi(v, t)=\frac{2 k v}{\sigma^{2}\left(e^{k(T-t)}-1\right)}, \quad \lambda=-\frac{k \Theta}{\sigma^{2}}+\frac{(1-\delta) \mu}{\rho \sigma}, \quad \eta=\sqrt{\left(\lambda+\frac{1}{2}\right)^{2}+\frac{2 C}{\sigma^{2}}},
$$

$M_{\lambda, \eta}(z)$ is a Whittaker's function, and $\Gamma(z)$ is a Gamma function.

Moreover, we have

$$
\frac{f_{v}}{f}=\frac{(\eta+\lambda+1 / 2)}{v} \frac{M_{1+\lambda, \eta}(\Psi(v, t))}{M_{\lambda, \eta}(\Psi(v, t))} .
$$


Proof. Making substitutions

$$
\tilde{v}=\frac{2 k v}{\sigma^{2}}, \quad k(T-t)=\tilde{\tau}, \quad f(v, t)=e^{-\lambda \tilde{\tau}} \tilde{v}^{\lambda} e^{\tilde{v} / 2} h(\tilde{v}, \tilde{\tau})
$$

we get the following boundary value problem for $h$

$$
\begin{array}{r}
h_{\tilde{v} \tilde{v}}+\left(-\frac{1}{4}+\frac{1 / 4-\eta^{2}}{\tilde{v}^{2}}\right) h=\frac{1}{\tilde{v}} h_{\tilde{\tau}} . \\
h(\tilde{v}, 0)=\tilde{v}^{-\lambda} e^{-\tilde{v} / 2} .
\end{array}
$$

Let $G(\tilde{v} ; \zeta)$ be the Laplace transform of function $h(\tilde{v}, \tilde{\tau})$ with respect to $\tilde{\tau}$

$$
G(\tilde{v} ; \zeta)=\int_{0}^{\infty} e^{\zeta \tilde{\tau}} h(\tilde{v}, \tilde{\tau}) d \tilde{\tau}
$$

By denoting $\chi(\tilde{v})=\tilde{v}^{-1-\lambda} e^{-\tilde{v} / 2}$, one can see that

$$
G^{\prime \prime}+\left(-\frac{1}{4}-\frac{\zeta}{\tilde{v}}+\frac{1 / 4-\eta^{2}}{\tilde{v}^{2}}\right) G=-\chi(\tilde{v}) .
$$

If made homogeneous, equation (4.8) is a Whittaker equation. Whittaker functions $M_{-\zeta, \eta}(\tilde{v})$ and $W_{-\zeta, \eta}(\tilde{v})$ are two linearly independent solutions of a Whittaker equation. One can check by substitution that the formula below is the solution of equation (4.8)

$$
G(\tilde{v} ; \zeta)=\frac{\Gamma(1 / 2+\zeta+\eta)}{\Gamma(1+2 \eta)}\left(M_{-\zeta, \eta}(\tilde{v}) \int_{0}^{\tilde{v}} \chi(\varphi) W_{-\zeta, \eta}(\varphi) d \varphi+W_{-\zeta, \eta}(\tilde{v}) \int_{\tilde{v}}^{\infty} \chi(\varphi) M_{-\zeta, \eta}(\varphi) d \varphi\right) .
$$

To proceed further we use formula 6.669.4 from [9]

$$
\begin{array}{r}
\int_{0}^{\infty} e^{-\frac{1}{2}\left(a_{1}+a_{2}\right) t \cosh x} \operatorname{coth}^{2 \nu}\left(\frac{1}{2} x\right) I_{2 \mu}\left(t \sqrt{a_{1} a_{2}} \sinh x\right) d x=\frac{\Gamma\left(\frac{1}{2}+\mu-\nu\right)}{t \sqrt{a_{1} a_{2}} \Gamma(1+2 \mu)} W_{\nu, \mu}\left(a_{1} t\right) M_{\nu, \mu}\left(a_{2} t\right), \\
\operatorname{Re}\left(\frac{1}{2}+\mu-\nu\right)>0, \quad \operatorname{Re} \mu>0, \quad a_{1}>a_{2} .
\end{array}
$$

The above formula from [9] allows us to rewrite (4.9) as

$$
G(\tilde{v} ; \zeta)=\sqrt{\tilde{v}} \int_{0}^{\infty} \int_{0}^{\infty} \varphi^{-1 / 2-\lambda} e^{-\frac{\varphi}{2}-\frac{\tilde{v}+\varphi}{2} \cosh \psi} \tanh ^{2 \zeta}\left(\frac{\psi}{2}\right) I_{2 \eta}(\sqrt{\tilde{v} \varphi} \sinh \psi) d \varphi d \psi
$$

Applying 6.643.2 from [9]

$$
\int_{0}^{\infty} x^{\mu-\frac{1}{2}} e^{-\alpha x} I_{2 \nu}(2 \beta \sqrt{x}) d x=\frac{\Gamma\left(\mu+\nu+\frac{1}{2}\right)}{\Gamma(2 \nu+1)} \beta^{-1} e^{\frac{\beta^{2}}{2 \alpha}} \alpha^{-\mu} M_{-\mu, \nu}\left(\frac{\beta^{2}}{\alpha}\right), \quad \operatorname{Re}\left(\mu+\nu+\frac{1}{2}\right)>0
$$

to the internal integral in (4.10), we obtain

$$
G(\tilde{v} ; \zeta)=e^{-\tilde{v} / 2} \frac{\Gamma(\eta-\lambda+1 / 2)}{\Gamma(1+2 \eta)} \int_{0}^{\infty} e^{(1-\cosh \psi) \tilde{v} / 4} \tanh ^{2 \zeta}\left(\frac{\psi}{2}\right)\left(\frac{\cosh \psi+1}{2}\right)^{\lambda} M_{\lambda, \eta}\left(\frac{\cosh \psi-1}{2} \tilde{v}\right) \frac{2 d \psi}{\sinh \psi} .
$$

By changing the variable of integration $\psi$ in (4.12) as

$$
2 \log \left[\tanh \left(\frac{\psi}{2}\right)\right]=\nu, \quad \frac{2 d \psi}{\sinh \psi}=d \nu, \quad \cosh \psi-1=\frac{2 e^{\nu}}{1-e^{\nu}}
$$


we get

$$
G(\tilde{v} ; \zeta)=e^{-\tilde{v} / 2} \frac{\Gamma(\eta-\lambda+1 / 2)}{\Gamma(1+2 \eta)} \int_{-\infty}^{0} \exp \left\{-\frac{\tilde{v}}{2} \frac{e^{\nu}}{e^{\nu}-1}+\zeta \nu\right\}\left(\frac{1}{e^{\nu}-1}\right)^{\lambda} M_{\lambda, \eta}\left(\tilde{v} \frac{e^{\nu}}{e^{\nu}-1}\right) d \nu .
$$

Inverting the Laplace transform, and using substitutions (4.7), we recover the formula for $f(v, t)$

$$
f(v, t)=\frac{1}{2 \pi i} \frac{\Gamma(\eta-\lambda+1 / 2)}{\Gamma(1+2 \eta)} \int_{N-i \infty}^{N+i \infty} \int_{-\infty}^{0} \exp \left\{-\frac{\tilde{v}}{2} \frac{e^{\nu}}{e^{\nu}-1}\right\} e^{\zeta(\nu+\tilde{\tau})}\left(\tilde{v} \frac{e^{-\tilde{\tau}}}{e^{\nu}-1}\right)^{\lambda} M_{\lambda, \eta}\left(\tilde{v} \frac{e^{\nu}}{e^{\nu}-1}\right) d \zeta d \nu
$$

where $N$ is a number such that all residues of the integrand are to the right of it.

Using the well-known representation of Dirac function

$$
\frac{1}{2 \pi i} \int_{N-i \infty}^{N+i \infty} e^{z \zeta} d \zeta=\delta(z)
$$

and changing the order of integration in (4.13), we get

$$
f(v, t)=\frac{\Gamma(\eta-\lambda+1 / 2)}{\Gamma(1+2 \eta)} \int_{-\infty}^{0} \delta(\nu+\tilde{\tau}) \exp \left\{-\frac{\tilde{v}}{2} \frac{e^{\nu}}{e^{\nu}-1}\right\}\left(\tilde{v} \frac{e^{-\tilde{\tau}}}{e^{\nu}-1}\right)^{\lambda} M_{\lambda, \eta}\left(\tilde{v} \frac{e^{\nu}}{e^{\nu}-1}\right) d \nu
$$

Note, that $\tilde{\tau} \geq 0$. Thus, we can complement the range of integration in (4.14) to the whole line, and, using the definition of Dirac's function, namely $\int_{-\infty}^{\infty} \delta(\zeta-z) g(\zeta) d \zeta=g(z)$ for any continuous $g$, we get

$$
f(v, t)=\frac{\Gamma(\eta-\lambda+1 / 2)}{\Gamma(1+2 \eta)} \exp \left\{-\frac{\tilde{v}}{2} \frac{e^{\tilde{\tau}}}{e^{\tilde{\tau}}-1}\right\}\left(\tilde{v} \frac{e^{-\tilde{\tau}}}{e^{\tilde{\tau}}-1}\right)^{\lambda} M_{\lambda, \eta}\left(\tilde{v} \frac{e^{\tilde{\tau}}}{e^{\tilde{\tau}}-1}\right) .
$$

Finally, by reversing the change of variables (4.7), we receive the main formula (4.4). The expression (4.6) for $f_{v} / f$ is obtained by using differential rules for Whittaker functions (see [1]). $\triangle$

The following theorem is the analogue of the results from section 3 but for Heston's model. It gives an exact solution for the optimal control and Bellman function.

Theorem 4.1. For power utility 2.4) and process (2.1) the Bellman function (2.3) is given by

$$
J_{P}(w, x, v, t)=\frac{w^{\gamma}}{\gamma} f^{1 / \delta}(v, t)
$$

and the optimal control is

$$
\alpha_{P}^{*}(w, x, v, t)=\frac{w}{x(1-\gamma)}\left(\frac{\mu}{v}+\frac{\rho \sigma}{\delta} \frac{(\eta+\lambda+1 / 2)}{v} \frac{M_{1+\lambda, \eta}(\Psi(v, t))}{M_{\lambda, \eta}(\Psi(v, t))}\right),
$$

where

$$
\begin{aligned}
f(v, t) & =\frac{\Gamma(\eta-\lambda+1 / 2)}{\Gamma(2 \eta+1)} e^{-\Psi(v, t) / 2}(\Psi(v, t))^{\lambda} M_{\lambda, \eta}(\Psi(v, t)), \\
\delta & =1+\rho^{2} \frac{\gamma}{1-\gamma}, \quad \lambda=-\frac{k \Theta}{\sigma^{2}}+\frac{(1-\delta) \mu}{\rho \sigma}, \quad C=-\frac{\gamma}{1-\gamma} \frac{\mu^{2}}{2} \delta, \quad \eta=\sqrt{\left(\lambda+\frac{1}{2}\right)^{2}+\frac{2 C}{\sigma^{2}}}, \\
\Psi(v, t) & =\frac{2 k v}{\sigma^{2}\left(e^{k(T-t)}-1\right)},
\end{aligned}
$$

$M_{\lambda, \eta}(z)$ is a Whittaker's function, and $\Gamma(z)$ is a gamma function. 
Theorem 4.2. For exponential utility 2.4) and process (2.1) The Bellman function is given by

$$
J_{E}(x, w, v, t)=1-\frac{e^{-c w}}{c} f^{1 / \delta}(v, t)
$$

and the optimal control is

$$
\alpha_{E}^{*}(w, x, v, t)=\frac{1}{c x}\left(\frac{\mu}{v}+\frac{\rho \sigma}{\delta} \frac{(\eta+\lambda+1 / 2)}{v} \frac{M_{1+\lambda, \eta}(\Psi(v, t))}{M_{\lambda, \eta}(\Psi(v, t))}\right),
$$

where

$$
\begin{aligned}
f(v, t) & =\frac{\Gamma(\eta-\lambda+1 / 2)}{\Gamma(2 \eta+1)} e^{-\Psi(v, t) / 2}(\Psi(v, t))^{\lambda} M_{\lambda, \eta}(\Psi(v, t)), \\
\delta & =1-\rho^{2}, \quad \lambda=-\frac{k \Theta}{\sigma^{2}}+\frac{(1-\delta) \mu}{\rho \sigma}, \quad C=\frac{\mu^{2}}{2} \delta, \quad \eta=\sqrt{\left(\lambda+\frac{1}{2}\right)^{2}+\frac{2 C}{\sigma^{2}}}, \\
\Psi(v, t) & =\frac{2 k v}{\sigma^{2}\left(e^{k(T-t)}-1\right)},
\end{aligned}
$$

$M_{\lambda, \eta}(z)$ is a Whittaker's function, and $\Gamma(z)$ is a gamma function.

Comment. The model (3.1) with drift $\mu\left(X_{t}, t\right)=V_{t}$ is studied in 11. For that particular model the coefficient for $f$ in (3.4) is proportional to $v$. This is the reason why the affine representation is possible in that specific case. Moreover, if the boundary condition is not constant, the affine representation is not possible again. Our method, on the contrary, allows us to obtain the required solution for any boundary conditions and for both models, either Heston's or the model considered in [11.

\section{Analysis}

\subsection{Analysis of the optimal control}

Optimal control in (4.17) and (4.19) is proportional to the sum of two terms: the first term $\mu / v$ corresponds to the static portfolio optimization problem. It it simply the ratio of the instantaneous drift to the instantaneous variance and does not depend on time or on the parameters of the volatility process. The second term

$$
\frac{\rho \sigma}{\delta} \frac{(\eta+\lambda+1 / 2)}{v} \frac{M_{1+\lambda, \eta}(\Psi(v, t))}{M_{\lambda, \eta}(\Psi(v, t))}
$$

represents the hedging of the future opportunity set ( see Merton [13]). This term vanishes in a number of important special cases:

$\rho=0$ : no correlation between the two driving Brownian motions,

$\gamma=0$ : when the investor has log-utility.

The case of zero volatility of volatility limit of Heston model ( $\sigma=0$ in (2.1) $)$ turns the asset process into a process similar to a geometric Brownian motion but with a deterministically time varying volatility. If in addition we set $V_{0}=\Theta$ in (2.1), then we get a geometric Brownian motion.

Using the asymptotic results from Appendix 6 in case $\sigma \sim 0$, we get

$$
f(v, t) \sim 1, \quad \frac{f_{v}(v, t)}{f(v, t)} \sim \frac{C}{k v^{2}} e^{k(T-t)} .
$$


Therefore, for $\sigma \sim 0$ the Bellman function is approximately equal to the utility function, namely $J_{P} \sim U_{P}, J_{E} \sim U_{E}$; and the optimal controls are

$$
\begin{aligned}
& \alpha_{P}^{*}(w, x, v, t)=\frac{w}{x(1-\gamma)}\left(\frac{\mu}{v}+\frac{\rho \sigma}{\delta_{P}} \frac{C_{P}}{k v^{2}} e^{k(T-t)}\right), \\
& \alpha_{E}^{*}(w, x, v, t)=\frac{1}{c x}\left(\frac{\mu}{v}+\frac{\rho \sigma}{\delta_{E}} \frac{C_{E}}{k v^{2}} e^{k(T-t)}\right),
\end{aligned}
$$

where constants $C_{[-]}$and $\delta_{[-]}$depend on the choice of utility.

\subsection{Hedging interpretation via a bond}

Suppose $C$ is given by (4.2) or (4.3). With substitution $C / v=r, f(v, t)=g(r, t)$ we can rewrite (4.1) as

$$
\begin{array}{r}
\frac{b^{2} r^{3}}{2} g_{r r}+h r(m-r) g_{r}-r g+g_{t}=0, \\
g(r, T)=1,
\end{array}
$$

where

$$
b=\frac{\sigma}{\sqrt{C}}, \quad h=-\frac{\sigma^{2}}{C}(1+\lambda), \quad m=-\frac{k C}{\sigma^{2}(1+\lambda)} .
$$

Using the Feynman-Kac formula we get the following expression for $g=g(r, t)$

$$
g(r, t)=\mathbf{E} e^{-\int_{t}^{T} r_{s} d s}
$$

where process $r=\left(r_{s}\right)_{s \geq t}$ is a solution to the following stochastic differential equation

$$
d r=h r(m-r) d t+b r^{3 / 2} d B^{2}, \quad r_{t}=C / v_{t} .
$$

Equation (5.6) describes the well-known 3/2-model of stochastic interest rates (see [8.) In this model, if $r$ is interpreted as the short rate, value of a zero coupon bond is given by (5.6), see [3]. Hence, the ratio $f_{v} / f$ can be expressed as

$$
\frac{f_{v}(v, t)}{f(v, t)}=\frac{d}{d v} \log \mathbf{E}\left[e^{-\int_{t}^{T} r_{s} d s} \mid r_{t}=C / v\right] .
$$

Substituting (5.7) into formulae for optimal control (4.17) and (4.19) one can get an interpretation of the hedging of the future opportunity set via a bond.

\section{Appendix. Technical asymptotics relations}

It follows from (4.4), that function $f=f(v, t)$ can be written as a function of only $\Psi$, namely $f=f(\Psi)$, where $\Psi=\Psi(v, t)$ is given by (4.5). This representation allows us to study the asymptotics of Bellman function and the optimal control by using the asymptotics of Whittaker functions $M_{\lambda, \mu}(z)$ (see, for example, [1]).

Indeed, by using the well known formulae below

$$
M_{\lambda, \eta}(z) \sim z^{\eta+1 / 2}(1+O(z)), \quad z \rightarrow 0, \quad M_{\lambda, \eta}(z) \sim \frac{\Gamma(1+2 \eta)}{\Gamma(1 / 2-\lambda+\eta)} e^{z / 2} z^{-\lambda}, \quad z \rightarrow \infty,
$$

we get

$$
f(v, t) \sim \frac{\Gamma(1 / 2-\lambda+\eta)}{\Gamma(1+2 \eta)} \Psi^{\eta+1 / 2}(v, t)\left[\Psi^{\lambda}(v, t)+O(\Psi(v, t))\right], \quad \Psi(v, t) \rightarrow 0,
$$


and

$$
f(v, t) \sim 1, \quad \Psi(v, t) \rightarrow \infty .
$$

For $f_{v}(v, t) / f$ we have

$$
\frac{f_{v}(v, t)}{f(v, t)} \sim \frac{(\eta+\lambda+1 / 2)}{v}, \quad \Psi(v, t) \rightarrow 0,
$$

and

$$
\frac{f_{v}(v, t)}{f(v, t)} \sim \frac{2 C}{\sigma^{2}} \frac{1}{v \Psi(v, t)}, \quad \Psi(v, t) \rightarrow \infty .
$$

Acknowledgements. The authors are grateful to Yury Kabanov for fruitful discussions and useful suggestions.

\section{References}

[1] M. Abramowitz, I. Stegun (1972) Handbook of Mathematical Functions with Formulas, Graphs and Mathematical Tables, Dover, New York.

[2] M. Boguslavsky, E. Boguslavskaya (2003) Arbitrage under power, RISK, 06, 49-53.

[3] D. Brigo, F. Mercurio (2006) Interest Rate Models - Theory and Practice: with Smile, Inflation and Credit, Springer Finance.

[4] G. Chacko and L. Viceira (2005) Dynamic consumption and portfolio choice with stochastic volatility in incomplete markets, Review of Financial Studies, 18, 1369-1402.

[5] J.C. Cox, J.E. Ingersoll, S.A. Ross (1985) A theory of the term structure of interest rates, Econometrica, 53, 2, 385-407.

[6] J. Fouque, R. Sircar and T. Zariphopoulou (2013) Portfolio optimization and stochastic volatility asymptotics, Preprint

[7] S. Heston (1993) A closed form solution for options with stochastic volatility with applications to bond and currency options. Review of Financial Studies, 6, 2, 327-343.

[8] J. Gatheral, N. Taleb (2006) Volatility Surface: a Practioner Guide, Wiley Finance.

[9] Gradshteyn I.S., Ryzhik I.M. (1980) Tables of Integrals, Series and Products, Academic Press, New York.

[10] Yu. Kabanov, M. Safarian (2009) Market with Transaction Costs. Mathematical Theory, Springer-Verlag.

[11] H. Kraft (2005) Optimal portfolios and Heston's stochastic volatility model: an explicit solution for power utility, Quantitative Finance, 5, 303-313.

[12] R.C. Merton (1971) Optimum consumption and portfolio rules in a continuous-time model, Journal of Economic theory, 3, 4, 373-413.

[13] R.C. Merton (1990) Continuous-Time Finance, Blackwell Publishers.

[14] A. Obizhaeva, J. Wang (2013) Optimal trading strategy and supply/demand dynamics, Journal of financial markets, 16, 1-32.

[15] T. Zariphopoulou (2001) A solution approach to valuation with unhedgeable risks, Finance and Stochastics, 5, 61-82. 